Cahiers de recherches médiévales

\title{
La fonction de la croisade dans Jehan de Saintré
}

Jane H. M. Taylor

\section{(2) OpenEdition}

Journals

Édition électronique

URL : https://journals.openedition.org/crm/2529

DOI : $10.4000 / \mathrm{crm} .2529$

ISSN : 1955-2424

Éditeur

Honoré Champion

Édition imprimée

Date de publication : 15 janvier 1996

Pagination : 193-204

ISSN : 1272-9752

Référence électronique

Jane H. M. Taylor, «La fonction de la croisade dans Jehan de Saintré », Cahiers de recherches

médiévales [En ligne], 1 | 1996, mis en ligne le 30 novembre 2009, consulté le 15 décembre 2022. URL : http://journals.openedition.org/crm/2529 ; DOI : https://doi.org/10.4000/crm.2529 


\section{La fonction de la croisade dans Jehan de Saintré}

Les longueurs - voire les ennuis - du Petit Jehan de Saintré sont de notoriété publique. Les longs sermons de Madame des Belles Cousines, les interminables faits d'armes auxquels se livre Saintré et, pis encore, la liste combien indigeste des croisés et de leurs armoiries ont peut-être su, se dit-on, intéresser le lecteur médiéval ; pour le lecteur du XXe siècle, ce sont généralement des parenthèses qu'il convient plutôt de sauter et où la plupart des critiques voient des maladresses de la part d'Antoine de La Sale.

Dans deux articles fort intéressants, toutefois, Madeleine Jeay nous a depuis peu invités à une réévaluation'. Pour elle, loin de trahir des inadvertances d'auteur, cette hétérogénéité du texte partirait d'un principe très établi de composition littéraire qui ne se veut pas romanesque, et où la thésaurisation, la mise en recueil, mieux, la mise en scène du récit, jouent un rôle primordial. On n'a qu'à voir avec elle le soin qu'Antoine de La Sale apporte à l'agencement sur la page des citations d'autorités ou des listes de participants dans la croisade de Prusse, pour comprendre que ces éléments digressifs en apparence sont au contraire fondamentaux à la conception que La Sale se fait de son ouvrage ${ }^{2}$. La dimension encyclopédique qui risque de nous paraître rébarbative serait au contraire la mission même de l'ouvrage, où la narration (qui d'ailleurs n'occupe qu'un petit tiers) serait en quelque sorte prétexte, et non finalité. Grâce aux aperçus de Jeay, il faudra donc reprendre l'étude du Petit Jehan de Saintré, à nouveau se pencher justement sur les longueurs du texte, revoir à travers la «multiplicité des langages» de celui-ci la réconciliation de la narrativité et de la description. Inspirée par elle, je voudrais dans ce qui suit réexaminer la longue description de la croisade et essayer de voir à travers son agencement textuel sa fonction thématique.

Mais d'abord, quelle croisade? Knudson, dans l'une des rares études dédiées à cette partie de l'œuvre ${ }^{3}$, a montré que toute fantaisiste qu'elle soit dans les détails, elle est fondée sur la réalité historique de ce XIVe siècle où l'authentique Saintré a évoluét. Il s'agit en effet d'une des nombreuses croisades en Prusse qui permettaient

\footnotetext{
1 «Les éléments didactiques et descriptifs de Jehan de Saintré : des lourdeurs à réconsidéren», Fifteenth-Century Studies, 19, 1992, pp. 85-100, et «Une théorie du roman: le manuscrit autographe de Jehan de Saintré», Romance Philology, 47/3, 1993-4, pp. 287-307.

${ }^{2}$ Selon la belle formule de Madeleine Jeay, «La splendeur du déploiement des marques sur la page. lorsqu'il s'agit des troupes chrétiennes, est bien destinée à mimer celle de ces troupes sur la champ de bataille» («Une théorie», p. 296).

${ }^{3}$ Voir Charles A. Knudson, «The Prussian Expedition in Jehan de Saintré», Etudes de langue et de littérature du moyen âge offertes à Félix Lecoy, Paris, Champion, 1973, pp. 271-77.

${ }^{4}$ Sur l'historicité de Saintré, voir entre autres Charles A. Knudson, «The Two Saintrés», Romance Studies in Memory of Edward B. Ham, éd. U.T. Holmes, Califomia State College Publications 2, Hayward, Ca., 1967, pp. 73-80, et id., "The Historical Saintré», Jean Misrahi
}

Cahiers de Recherches Médiévales (XIIT-XV s.), 1, 1996 
au chevalier désœuvré de se faire une réputation chevaleresque ${ }^{5}$. Le roi de France, Jean le Bon, recrute une armée remarquable qu'il met sous les ordres du jeune écuyer Jehan de Saintré qui, grâce à ses prouesses dans les emprises et les faits d'armes, a déjà acquis une retentissante réputation européenne. Le corps expéditionnaire envahit donc la Prusse et se réunit à «Torrin» (polonais Torun, allemand Thorn) avec des détachements venus de tous les coins de l'Empire, sous les ordres du «roy de Behaigne en personne». Les Chrétiens vont se mesurer avec «la plus grant armee que depuis la loy de Mahommet [les Sarrasins] eussent faite» (JS, pp. 212/33-213/1). Saintré lui-même sera fait chevalier par le roi de Bohème et aura le bonheur au cours d'une glorieuse victoire de tuer de sa propre main le Grand Turc. Il revient donc en France comblé d'honneurs et au sommet d'une gloire chevaleresque.

Il va sans dire que cet insigne triomphe n'a que peu à voir avec la réalité. S'il est vrai que les chevaliers français s'aventuraient régulièrement en Prusse, il reste qu'ils n'ont jamais remporté une victoire aussi éclatante - mieux même, les croisades de ce siècle essuyaient plutôt des échecs, à commencer par celui, lamentable, de Nicopolis. Cela dit, le lecteur du XV siècle qui parcourait Jehan de Saintré n'a pas dû trouver incongru le fait que la croisade reste l'aventure la plus méritoire où un jeune chevalier puisse se risquer ${ }^{7}$ et aurait sans doute feuilleté avec un plaisir légèrement teinté de nostalgie et de regret des pages où une armée majoritairement française et commandée par un jeune Français remportait une victoire aussi éblouissante sur les Infidèles. Que ce dernier voit sa carrière triomphante de chevalier «errant» couronnée par un adoubement sur le champ de bataille, devant les Infidèles, et par la main de celui qui en quelque sorte représentait une chevalerie parfaite mais révolue ${ }^{8}$, ne devait avoir rien de surprenant : après tout, en 1396, le comte de Nevers fut envoyé

Memorial Volume: Studies in Medieval Literature, éd. Hans R. Runte, Henri Niedzielski, William L. Hendrickson, Columbia, S. C., French Literature Publications, 1977, pp. 248-309. ${ }^{5}$ Au sujet des croisades en Prusse, voir entre autres A.S. Atiya, The Crusade in the Later Middle Ages, London, Methuen, [1938] ; id., The Crusade of Nicopolis, London, Methuen, 1934 ; Eric Christiansen, The Northern Crusades: The Baltic and the Catholic Frontier 1100-1525, New Studies in Medieval History, London/Basingstoke, Macmillan, 1980 (surtout p. 132 ss : «The Interminable Crusade», 1283-1410).

${ }^{6} \mathrm{Je}$ cite d'après l'édition la plus connue, celle de Jean Misrahi et de Charles A. Knudson, Jehan de Saintré, Genève, Droz, 1978, p. 211 (citations désormais dans le texte, précédées du sigle $J S$. Une nouvelle édition d'après le manuscrit Barrois (BN fonds fr. 10057) est due aux soins de Mario Eusebi, Paris, Champion, 1993.

${ }^{7}$ Que l'on pense, par exemple, aux projets de croisade régulièrement annoncés au cours du quinzième siècle, et notamment aux Voeux du Faisan; voir R. Vaughan, Philip the Good: The Apogee of Burgundy, London, Longman, 1970, p. 358ss.

${ }^{8}$ On sait quelle était la réputation du roi de Bohème au XIVe et au XVe siècle. Froissart par exemple voyait en lui une réplique d'Alexandre le Grand et des autres Preux : «Li bons rois,/Qui tant fu larges et courtois/Que, de Prusse jusqu'en Artois,/Non, jusques en Constantinnoble/N'i eut plus large ne plus noble» (Prison amoureuse, éd. A. Fourrier, Paris, 1974, vv. 65-69). Voir à ce sujet Peter Dembowski, Jean Froissart and his Meliador: Context, Craft, and Sense, Lexington, Kentucky, French Forum Publishers, 1983, p. 48ss. 
précisément en Prusse par son père Philippe de Bourgogne, qui voulait que son fils gagnât ses éperons de chevalier en combattant lui aussi contre les Infidèles?.

D'un côté donc, cet épisode marquerait tout simplement l'apogée d'une carrière-type de jeune chevalier destiné à devenir "[le] plus vaillant chevalier de France, et plus, que pour lors sera» $\left(J S\right.$, p. 308/29-30) ${ }^{10}$. Mais cela n'expliquerait pas pourquoi la description de la croisade est si complaisamment, si démesurément prolongée. A quoi servent ces longues listes de participants, leur blason, leur cri de guerre? A première vue, on est tenté de supposer, avec Knudson, que La Sale recopierait sans arrière-pensée éditoriale un document héraldique ${ }^{11}$ ayant trait à une authentique expédition de croisés ; autrement dit, on aurait affaire à un emprunt. Mais précisons: les fameuses listes partent d'un considérable travail de reconstruction pseudo-historique dans la mesure où aucune croisade du XIVe siècle, aucun rassemblement du XV $V^{e}$, n'a regroupé une armée d'une telle envergure. Même si La Sale a pu disposer de documents existants, il a dû trier, sélectionner ${ }^{12}$ : ces listes comprennent non seulement des combattants français - pour lesquels sans doute des armoriaux étaient à portée de main - mais des forces anglaises, allemandes, impériales... Il lui a fallu aussi inventer une horde de «Sarrasins» venus de partout d'«Ayse la maiour», «de Persse», «de Surie» (JS, p. 213) - et qu'il a voulu documenter avec des informations solides et exactes : ainsi sont mises à notre disposition des précisions géographiques et historiques, héraldiques (de la propre invention de l'auteur $^{13}$ ?), voire bibliques ${ }^{14}$. Il ne s'agit donc pas d'un vulgaire plagiat, mais plutôt d'une véritable esthétique de la thésaurisation, d'un encyclopédisme que l'on sent réfléchi.

D'ailleurs, l'épisode de la croisade n'est pas fait que des listes. Au contraire, on ressent à la lecture de ces pages une véritable recherche stylistique qui laisse sans

\footnotetext{
9 Voir Delaville Le Roulx, La France en Orient au quatorzième siècle. Expédition du maréchal Boucicaut, Paris, E. Thorin, 1886, t. I, pp. 233-234, et Denis Lalande, Jean II le Meingre, dit Boucicaut (1366-1421); étude d'une biographie héroíque, Genève, Droz, 1988, p. 58.

${ }^{10}$ Notons que c'est précisément d'un destin qu'il s'agit, puisque c'est en creusant sa sépulture que les fossoyers trouvent déjà en place «un petit escrinet ouquel avoit un brevet qui disoit : Cy reposera ...» (JS, p. 308/27-29).

${ }^{11}$ Knudson, «Saintré's Prussian Expedition». Cette hypothèse n'est pas à écarter, bien que ni les recherches de Knudson ni celles de Max Prinet n'aient pu la confirmer.

${ }^{12}$ La banalité du topos ne doit d'ailleurs pas nous faire ignorer les fréquentes remarques métatextuelles qui témoignent précisément de ce souci éditorial : «desquelz pour abregier je me passe» (JS, 212/25) ; «que pour abregier je m'en passe» (JS, p. 221/4); et surtout une longue explication (JS, p. 218/25-30) : «et de les nommer seroit tres longue chose et de decliner leurs proesses ; aussi qui ne feroit declaracion des armes des ungs comme des autres, j'en pourroye estre en male grace ; par quoy je prie a tous que a tant leur veulle souffire et soye tenu pour excusé».

${ }^{13}$ Aurait-il trouvé ou inventé par exemple ses informations sur les armoiries d'«Abzin le Grant Turcq de Persse» ou de «Zisaach», «qui se disoit empereur de Cartaige» (voir $J S$, p. 216)?

14 Ainsi de la «mer noire»: La Sale (JS, p. 213) nous renseigne sur le climat, les principales îles et cités, la faune ( $\mathrm{y}$ multiplient plus les olifans que en autre partie du monde»), l'histoire religieuse («convertie par saint Thomas l'apostre»)....
} 
doute deviner l'importance que La Sale devait attacher à ces pages à première vue ingrates. Y figurent des fleurs de rhétorique que l'on retrouve rarement ailleurs dans le texte: des doublets et des triplets synonymiques («la tres fiere, cruelle et mortelle bataille» [JS, p. 220/13]; «les mors a tresgrans honneurs et plains et a solempnelz services de Dieu furent enterrez» [JS, p. 221/20-22]), des inversions du sujet faites à des fins expressives («La fu d'eulz ]'occision si grande...» [JS, p. 220/29-30]), de dramatiques anaphores ( $J S$, p. 220/29-32), des comparaisons éloquentes («sans plus de deffense con se fussent brebis» [JS, p. 220/21-22] ), des expressions superlatives où transperce l'émotion de l'écrivain («chiennaille de gens maudiz» [JS, p. 220/19]). Cet épisode que nous aurions tendance aujourd'hui à laisser de côté a donc été travaillée, peaufinée; il s'agit - dirait-on - de la recherche d'une «poéticité».

Mais à quelle fin? Si le long épisode de la croisade a été à ce point élaboré, il faut supposer que cela ressortit non seulement d'une esthétique de l'écriture, mais aussi d'une conception narrative arrêtée, et d'une conception ancrée dans ce qui constitue tout de même, et malgré une mission pédagogique évidente, la trame narrative de l'œuvre, les amours de Saintré et de Madame des Belles Cousines. Comment donc voir dans la croisade, étape définitive pour le chevalier-type du XVe siècle, un épisode fondamental de la biographie amoureuse de ce jeune homme qui, lui, en est l'amant-type ? On sait - je l'ai d'ailleurs déjà indiquée ${ }^{15}$ - combien la croisade et la reconquête de la ville sainte sont restées dans la conscience idéologique de l'Europe un rêve à réaliser, et on ne saurait sous-estimer l'importance de la croisade dans la vie «fictionnelle» de ce siècle. C'est la croisade, en effet, qui constitue en quelque sorte l'apothéose d'un Cleriadus ${ }^{16}$, ou d'Ysaÿe le Triste et de son fils Marc ${ }^{17}$; qui sous-tend ce curieux roman de la fin du XV $\mathrm{XV}^{\mathrm{e}}$ siècle, Le roman de messire Charles de Hongrie ${ }^{18}$; qui permet à Jean d'Avesnes d'acquérir enfin les bonnes grâces de la comtesse d'Artois ${ }^{19}$. Mieux encore, la chanson de geste, patrie s'il en est de l'esprit de la croisade, revit sous la forme des mises en proses ${ }^{20}$; une partie du Cycle de la Croisade, par exemple, est refaite pour la cour de Bourgogne $e^{21}$, et c'est un souci majeur de David Aubert, dans sa grande compilation Les Chroniques et conquestes de Charlemaine ${ }^{22}$, que de glorifier le Charlemagne conquérant du monde arabe. Lorsque donc, dans La Salade ${ }^{23}$, La Sale préconise pour l'écuyer ambitieux un adoube-

\footnotetext{
${ }^{15}$ Voir à la note 7, supra.

${ }^{16}$ Cleriadus et Meliadice, éd. Gaston Zink, Genève/Paris, Droz, 1984, ch. xxi, pp. 283-90.

${ }^{17}$ Voir Ysaÿe le Triste, roman arthurien du Moyen Age tardif, éd. A. Giacchetti, Publications de l'Université de Rouen, Rouen, 1989, §621.

${ }^{18}$ Ed. Marie-Luce Chênerie, Toulouse, Presses Universitaires du Mirail, 1992. Pour l'éditrice, «les correspondances et les repères internes se multiplient avec Charles VIII» (p. xxvi).

${ }^{19}$ Voir Jehan d'Avesnes, éd. Anna Maria Finoli, Milan, Cisalpino-Goliardica, 1979, p. 173.

${ }^{20}$ Voir par exemple les remarques de Georges Doutrepont, Les Mises en prose des épopées et des romans chevaleresques du XIVe au XVI siècle, Bruxelles, Palais des Académies, 1939, p. 398ss.

${ }^{21}$ Voir Saladin, éd. Larry S. Crist, Genève, Droz, 1972, pp. 9-15.

${ }^{22}$ Voir à ce sujet Georges Doutrepont, op. cit., pp. 63-86.

${ }^{23}$ Cuures complètes, t. I : La Salade, éd. Fernand Desonay, Liège/Paris, Droz, 1935, p. 234 : «L'escuier, quant il a bien voyagé et a esté en pluiseurs fais d'armes, dont il en est sailly a honneur... ; dont, pour plus honnorablement estre [chevalier], que avant la battaille, l'assault ou le rencontre ou bannyeres de princes soient, alors doit requerir aucum seigneur ou preu-
} 
ment très chrétien sur le champ de bataille, il devait sans doute avoir en tête un cadre comme celui qu'il fournit à son héros Saintré.

Mais qu'en est-il - répétons-le - des amours de celui-ci? Faut-il voir dans la croisade une contribution à une œuvre de compilation comme il y en a tant au XVe siècle, ou possède-t-elle une fonction narrative dans le «toman» de Saintré et de Madame $^{24}$ ? Il me semble - et ce sera l'essentiel de ma démonstration - que l'importance de la croisade en Prusse réside dans sa fonction de point de bascule dans le jeu complexe des relations de pouvoir entre Saintré et Madame, fonction narrative manifestée, on le verra, à travers certaines formules et expressions récurrentes. Le Saintré qui a évolué et qui est resté jusque là dans un monde régi par le pouvoir quasi-«maternel» de Madame, et qui a donc été en quelque sorte infantilisée $e^{25}$, sera transposé dans un monde exclusivement et résolument masculin évoqué précisément par les listes des participants, par «l'altérité» de la croisade; il en reviendra prêt à assumer une identité et une autonomie masculines.

Rappelons d'abord la façon dont La Sale conjugue les deux fils conducteurs pour amener dans la trame romanesque l'épisode de la croisade. Saintré, on le sait, s'est déjà mesuré contre une suite d'adversaires dans des pas d'armes, et en est sorti triomphant. La Sale s'attarde complaisamment sur sa réputation :

Estant Saintré... le plus amé, le plus honnoré escuier de France à cause de sa grant doulceur et humilité, et aussi de sa largesse, qui aide bien au jeu. Car onques pour gloire d'amour de roy ne d'autre ne d'onneur qu'il eust, un seul semblant d'orgueil ne fut en luy. ( $J S$, pp. 186/32-187/6)

Il est donc au comble d'une célébrité «de cour» lorsque la croisade de Prusse est annoncée. Comme à chaque occasion jusqu'ici, c'est Madame qui va déterminer en quelque sorte sa carrière de chevalier - et dans des termes significatifs :

Mon seul desir et toute ma pensee, tant est l'amour saine et entiere que j'ay en vous pour vous faire le meilleur et le plus vaillant homme du monde que vraiement elle estaint de mon cuer la doubteuse crainte que j'ay et dois avoir de vous. Maiz, pour ceste foiz seullement et non plus, vous y veul adventurer. (JS, pp. 187/12-18)

Ceci reprend bien sûr et avec un écho textuel l'objectif que Madame s'était proposé dès le début de sa relation avec Saintré : «Ceste dame... se pensa qu'elle vouloit

domme chevalier qu'il le face chevalier ou nom de Dieu, de Nostre Dame et de monseigneur saint George, le bon chevalier, en lui baillant son espee nue en baisant la croix.» Voir à ce sujet mon article "The Pattern of Perfection : Jehan de Saintré and the Chivalric Ideal", Medium Aevum, 53, 1984, 254-62.

${ }^{24}$ Il convient, me semble-t-il, de distinguer la partie proprement narrative du Petit Jehan de Saintré (soit le «roman») du «livre», ce dernier désignant ce qu'on pourrait appeler l'œuvre dans sa totalité où figurent, à côté de la narration, les éléments à mission pédagogique ou inspiratrice.

${ }^{25} \mathrm{Qu}$ 'on me passe cette expression peut-être saugrenue. J'entends par là que Madame, autoritaire et même en quelque sorte maternelle, empêche le jeune Saintré de se faire une identité chevaleresque et même masculin, prolonge démesurément son adolescence, malgré ce qui semble être une maturité sexuelle. 
en ce monde faire d'aucun josne chevalier ou escuier un homme renommé» (JS, p. 6. 1-7), comme d'ailleurs la réponse de Saintré à la proposition de Madame: «A! ma tresnoble et souveraine deesse, celle qui me puet et doit plus commander, et celle qui a cui je doy et veul plus obeï que a tout le demeurant du monde.... auquel vostre vouloir... je obeiray» ( $S S$, p. 188/3-10) correspond à sa réponse d'adolescent devant la dame qui se propose à lui : «Madame, je feroie tout ce que me vouldriés commander» (JS, p. 36/14-15). Mais l'époque où Saintré était un jeune écuyer de treize ans, terrorisé par Madame et ses suivantes, est bien révolue; ne faudrait-il pas voir dans la répétition de cette formule, et malgré les protestations d'amour de Madame, les signes d'une infantilisation tant soit peu gênante pour celui qui est d'ores et déjà le champion chevaleresque de la France et à qui sera confié le commandement de l'armée française? Une autre reprise textuelle semblerait l'indiquer, car Madame réemploie un verbe que nous avons appris à connaître: «Vous y veul adventurer» «Nous voulons que vous y alez en grant estat.» (JS, p. 187/32-33) Une formule analogue ( veul et vous commande ») a notamment rythmé les premières tirades de Madame $^{26}$, adressées à l'adolescent dont l'éducation était entièrement à faire, et avec une insistance remarquable mais qui ne semblait pas, dans cette situation, trop inconvenante. Plus tard - et là la répétition semble déjà un peu plus inattendue - elle a scandé les faits d'armes de Saintré27. Saintré est resté, si l'on peut dire, objet de phrase: toujours soumis au vouloir de celle qu'il s'est engagé - et là aussi les expressions sont significatives - à servir et à obéir : «Ma dame, celle qui me puet plus commander et que je doy et veul plus obeïn (JS, p. 69/22-23) - phrase reprise, ou peu s'en faut, en toutes lettres par Saintré lorsqu'il réagit à la proposition de Madame dans les mots que nous avons cités plus haut: «A! ma tresnoble et souveraine deesse, celle qui me puet et doit plus commander, et celle qui a cui [sic] je doy et veul plus obeir que a tout le demeurant du monde...» (JS, p. 188/3-6). Il existe donc si l'on peut dire un «contrat» ou «pacte» - obéissance /service contre amour/largesse - contrat qui se fait remarquablement explicite dans $J S$ : «Tant que me servirez loialment je vous fourniray du tout» (JS, p. 72/9-10) ; «Je qui vous ay pour moy servir sur toutes autres choisy, vous prie encores que ne vous sociez fors que de estre liez et joyeux, et par tout faire bonne chiere, car d'or, d'argent et de bagues pour vous mectre bien en point a ce vostre commencement, je vous fourniray assez» (JS, p. 80/1-6).

Le «contrat» - cette dépendance - a eu comme effet de retenir le héros résolument dans un monde régi par les «dames» ${ }^{28}$, fait souligné par l'expression «vous ad-

${ }^{26}$ Elle réapparaît 26 fois dans la bouche de Madame entre les pages 36 et 50 .

${ }^{27}$ Voir par exemple : «Je veul que pour l'amour de moy vous portez un bracelet d'ons (WS, p. 79/14-15) ; «Tant est l'amour que je vous porte que vous vouldroie en tous endrois le plus vaillant» (JS, p. 146/22-23); "Je veul que a l'ayde de Dieu... vous lui acomplissez» (JS, p. 174/12) .

${ }^{28}$ Dans un article à paraitre dans Medioevo Romanzo, «Courtly Patronage Subverted : Lancelot en prose, Petit Jehan de Saintré », j'ai voulu montrer que cette infantilisation du héros est à lire en contredistinction de la façon dont Guenièvre et la Dame du Lac «libèrent» en quelque sorte Lancelot ; voir à ce sujet aussi l'intéressant article de Françoise Paradis, «La triple mise au monde d'un héros, ou trois images d'une féminité maitrisée dans le début du Lancelot en prose", Approches du Lancelot en prose, éd. Jean Dufournet, Paris, Champion, 1984, pp. 157-76. 
venturer» qui trahit le peu d'autonomie du jeune chevalier. Je citais il y a quelques instants le panégyrique de Saintré qui se trouve placé juste avant l'annonce du "voiage de Prusse» - panégyrique où les qualités mises en évidence sont moins celles du guerrier que du chevalier servant ou du courtisan : amé, doulceur, humilité, largesse. La croisade lui permettra enfin de se dégager de ce monde «féminisé»" et de s'intégrer au monde masculin. On reconnaissait bien sûr à la fin du moyen âge quelle distance séparait le monde ludique du «jeu» chevaleresque ${ }^{30}$ dans le monde des femmes et celui de la dure réalité guerrière ${ }^{31}$ - témoin par exemple les paroles attribuées à Jean de Beaumont, dans les Voeux du héron:

Quant nous somme en tavernes, de ches fors vins boevant,

Et ches dames de l'es qui nous vont regardant,

A ches gorgues polies ches colieres tirant,

Chil œil vair resplendissant de beauté souriant,

Nature nous semont d'avoir ceur desirant

De contendre, à le fin de merchi atendant ;

Adonc conquerons nous Yaumont et Aguilant,

Et li autre conquirent Olivier et Rolant.

Mais quand sommes as camps, sor nos destriers courans,

Nos escus à nos cols, et nos lanches baissans,

Et le frodure grande nous va tous engelans,

$\mathrm{Li}$ membre nous effendent et derriereet devant,

Et nos ennemis sont envers nous approchant ;

Adonc vauriemes estre en un chelier si grant

Que jamais ne faissons veu ne tant ne quant. ${ }^{32}$

La croisade représente donc en quelque sorte la récupération du chevalier par le compagnonnage des hommes ${ }^{33}$. Elle représente peut-être aussi la réintégration du

29 J'ai étudié ailleurs la «féminisation» du monde chevaleresque de $J S$ : voir "The Parrot, the Knight and the Decline of Chivalry", Conjonctures: Medieval Studies in Honor of Douglas Kelly, éd. Keith Busby and Norris J. Lacy, Amsterdam, Rodopi, 1994, pp. 529-44 .

${ }^{30}$ Voir à ce sujet la remarquable étude de Michel Stanesco: Jeux d'errance du chevalier médiéval: aspects ludiques de la fonction guerrière dans la littérature du moyen âge flamboyant, Leiden, Brill, 1988.

31 Johann Huizinga aurait tendance, peut-être, à trop mettre l'accent sur les rêves de la chevalerie et de l'héroïsme (voir les chapitres 4 à 7 de son remarquable L'Automne du moyen âge). Pour un portrait plus juste du côté «guerrier» de la vie du chevalier errant, voir par exemple Maurice Keen, Chivalry, New Haven and London, Yale University Press, 1984, pp. 219-237, et Malcolm Vale, War and Chivalry, London, Duckworth, 1981, pp. 1-12. Pour une intéressante étude de cas, voir Maurice Keen, «Gadifer de La Salle : A late medieval knight errant», The Ideals and Position of Medieval Knighthood: Papers from the first and second Strawberry Hill Conferences, éd. Christopher Harper-Bill and Ruth Harvey, Woodbridge, Boydell and Brewer, 1986, pp. 74-85.

${ }^{32}$ Les Voeux du héron, éd. Thomas Wright, Political Poems and Songs Relating to English History composed during the period from the Accession of Edward III to that of Richard III, Rolls Series 14a, London, Longman Green Longman and Roberts, 1859, t. I, p. 21. Le dernier vers de la citation est faux, mais il en est ainsi dans l'édition.

${ }^{33}$ Sur l'importance du compagnonnage, voir entre autres Marie-Luce Chênerie, Le Chevalier errant dans les romans arthuriens en vers du XII siècle, Genève, Droz, 1986. 
héros dans l'histoire, autrement dit elle signifierait une rupture d'avec le monde romanesque du chevalier errant que Saintré a jusqu'ici habité. Sans vouloir distinguer avec une netteté anachronique roman et histoire ${ }^{34}$, il est à noter que ce n'est jamais avec le mot roman que La Sale désigne son œuvre (il préfère istoire, livre, traité ${ }^{35}$ ). Tout d'ailleurs laisse deviner une ambition d'historien, à commencer par la nature de l'ouvrage qu'il projetait de présenter à son patron: si La Sale a voulu, à l'origine, compiler avec Jehan de Saintré un long extrait voué aux guerres de Flandre ${ }^{36}$, on se demande si ce fait même n'indiquait pas que cette grande compilation remarquablement hétérogène avait une finalité plus documentaire que fictionnelle ${ }^{37}$. Les lecteurs qui voient dans les faits d'armes et dans la croisade une parenthèse dans le «roman" se trompent peut-être d'optique: ne faudrait-il pas plutôt voir dans le «roman» une parenthèse fâcheuse dans la biographie chevaleresque ${ }^{38}$, genre auquel Jehan de Saintré s'apparente vraisemblablement plus qu'au genre fiction. Le héros, après tout, agit en permanence en marge de l'histoire: au moment où la croisade est proclamée, il vient de confirmer sa réputation de futur guerrier en combattant contre les Anglais dans un pas d'armes qui rappelle instamment les Joutes de Saint Inglevert racontées par Jean Froissart ${ }^{39}$. En un sens, les longues listes de participants confirmeraient la transformation d'un héros au statut plutôt fictionnel en un héros historique à part entière, digne de figurer aux côtés de Jacques de Lalaing ${ }^{40}$ et de Boucicaut $^{41}$.

Rappelons aussi l'introduction de ce dernier personnage dans le texte de Jehan de Saintré ; en effet, peu avant de prendre la croix, Saintré a en un sens été accaparé par le monde masculin du compagnonnage. Le livre nous rend témoins des étapes

\footnotetext{
${ }^{34}$ Cette question extrêmement complexe et intéressante est traitée avec beaucoup de finesse par Ruth Morse, Truth and Convention in the Middle Ages: Rhetoric, Representation, and Reality, Cambridge etc., Cambridge University Press, 1991 ; voir surtout les pages 85-124.

${ }^{35}$ Istoire : JS, p. $1 / 6$; Livre: JS, p. 308/9, 309/22 ; traictiez : JS, p. $1 / 7$.

${ }^{36}$ L'extrait est publié par Yorio Otaka, Jehan de Saintré, suivi de l'Adicion extraicte des Croniques de Flandres, Tokio, Takeuchi, 1967, pp. 263-273.

${ }^{37}$ Remarquable, car La Sale s'était déjà largement exercé dans le métier de compilation : ses deux œuvres majeures, La Salade (1422-44) et La Sale (1451), sont des compilations pédagogiques et didactique, la première destinée aux enfants de Jean de Calabre, la deuxième dédiée à Louis de Luxembourg.

${ }^{38}$ Dans un article basé sur les manuscrits illustrés de Jehan de Saintré, j'ai voulu montrer que le témoignage iconographique semblerait indiquer que c'est ainsi que le roman a été reçu: voir «Image as Reception : Antoine de La Sale's Le Petit Jehan de Saintré », Literary Aspects of Courtly Culture: Selected Papers from the Seventh Triennial Congress of the International Courtly Literature Society, University of Massachusetts, Amherst, 27 July - 1 August 1992, éd. Donald Maddox and Sara Sturm-Maddox, Cambridge, D. S. Brewer, 1994, pp. 265-79.

${ }^{39}$ Voir Froissart, Chroniques, éd. Kervyn de Lettenhove, Bruxelles, Académie Royale de Belgique, 1867-77, t. XIV, p. 105ss.

${ }^{40}$ On sait qu'un passage de Jehan de Saintré (figurant dans le sermon de Madame, JS, pp. 17ss, a été recopié sur la biographie de Jacques de Lalaing ; voir Gaston Raynaud, «Un nouveau manuscrit du Petit Jehan de Saintré ", Romania, 31, 1902, pp. 527-556.

${ }^{41}$ Sur la relation entre biographie authentique et biographie fictionnelle au $\mathrm{XV}^{\mathrm{e}}$ siècle, et notamment à la cour de Bourgogne, voir Ruth Morse, «Historical Fiction in Fifteenth-Century Burgundy», Modern Language Review, 75, 1980, pp. 48-64.
} 
d'une évolution chevaleresque manifestée d'une part à travers une série progressive de combats singuliers, et d'autre part à travers un kaléidoscope de relations où l'histoire et la fiction se trouvent constamment imbriqués ${ }^{42}$. Ainsi Madame se dit nièce des «oncles» de la famille royale ${ }^{43}$, et ainsi l'action se déroule à l'authentique cour de Jean le Bon et de Bonne de Luxembourg ${ }^{44}$. Le Boucicaut de l'histoire se glisse donc dans le livre, en quelque sorte comme réplique de Saintré :

Ja soit ce que Boussicault fust puis tres vaillant chevalier, oultre plus estoit il plus soubtil et actrempé que Saintré n'estoit ; mais au fait des armes Saintré estoit tenu le plus avant. Et pour ce les roys d'armes et heraulz en firent un commun proverbe en disant :

Quant vient a un essault,

Mieulz vaut Saintré que Boussicault ;

Mais quant vient a un traicté,

Mieulz vaut Boussicault que Saintré.

C'est assavoir, l'un pour les armes, l'autre pour le conseil. Dont par ainsin, tant que ilz ensemble vesquirent, leur amour et leur estat dura. (JS, p. 143/6-18)

Cette relation en symbiose, où les qualités du chevalier parfait se trouvent partagées entre les deux héros - qui deviennent, selon la formule de La Sale, des «frères» $(J S$, p. $181 / 23$, p. $182 / 4,10)$ - est introduite dans le «roman» au moment même où Saintré semble avoir passé l'âge de se plier aux voeux et aux craintes de Madame. Dans leur première aventure, Saintré relève le défi du seigneur de Loisselench, qui, à l'effroi de Madame, s'avère être épouvantablement «haut et corpulent» (JS, p. 149/22-23). Madame est prise d'une crainte qui semblent témoigner d'un manque de confiance et où l'érotique et le maternel semble, comme c'est souvent le cas dans le "roman», inextricablement mêlés; dans une longue tirade Madame évoque non seulement l'amant («cellui que en ce monde plus amoyes [sic]») mais aussi le protégé qu'elle a élevé et formé («je l'aye conforté seullement pour estre entre les bons et les preux": $J S$, pp. 149/30-150/6-8). Il est vrai qu'elle reconnaît finalement que la renommée de Saintré exige qu'il se mesure contre le «jaiant qu'il est au regart de» Saintré (JS, p. 151/10), qu'elle l'envoie au combat avec cette aide financière qui lui aura permis de s'imposer en tant que champion ${ }^{45}$. Mais pour la première fois, on

\footnotetext{
12 Bien que de façon déconcertante : la vérité historique est -- à dessein ? -- déformée (comme l'a bien montré Denis Lalande : voir son article «Le couple Saintré-Boucicaut dans le roman de Jehan de Saintré ", Romania, 111, 1990, 481-94). Mais La Sale fait appel précisément à l'histoire ; voir les articles de Knudson (note 4, supra).

${ }^{43} J S$, p. 79/24-26 : «messeigneurs mes beaus oncles de Anjou, de Berry et de Bourgoigne, et autres seigneurs de nostre sang» (voir aussi $J S$, p. 89/26).

4 Notons toutefois qu'ici aussi, La Sale tient peu compte du détail historique: Bonne de Luxembourg est morte en 1349, et n'a donc jamais été reine.

45 "Dont, mon ami, ne vous soussiez que de bien faire, et quant au regart de vostre despense et de vous abillier honorablement, veez ci en ce saichet $\mathrm{vi}^{\mathrm{m}}$ escuz, et despendez honorablement et a Dieu soiez.» (US, p. 151/17-21) Ce curieux mélange du romanesque et du «commercial» est constant dans l'ouvrage : voir par exemple $J S$, pp. 49/31-50/13 ; pp. 69/4 19 etc. Voir à ce sujet l'intéressante étude d'Emma Stojkovic Mazzariol, L'Occhio e il piede; lettura critica del Petit Jehan de Saintré di Antoine de La Sale, Vicenza, Neri Pozza, 1979, p. 67 ss.
} 
verra Saintré non pas uniquement à travers sa relation avec Madame, mais plutôt fusionné avec un authentique champion masculin. Désormais, et de plus en plus, sa carrière se verra identifiée avec celle des Boucicaut. Or, comme on le sait, La Sale, à dessein ou non, a systématiquement confondu les deux Boucicaut ${ }^{46}$ : le père, qui fut authentiquement le compagnon d'armes de Saintré, avec le fils, Jean II le Meingre, objet d'une longue biographie chevaleresque et qui, lui, a notamment participé plusieurs fois à des croisades en Prusse.

A eux deux, les Boucicaut représentent ainsi l'histoire, le véridique - et il ne serait peut-être pas trop fort de parler d'un effet de métonymie: la présence de Boucicaut aux côtés du héros romanesque change profondément l'optique de l'ouvrage et le statut du héros. Alors que Madame (malgré son mystérieux anonymat et les "garanties» de $\mathrm{La} \mathrm{Sale}{ }^{47}$ ) représentait la fiction qui, en un sens, ancrait le Saintré historique au «roman», Boucicaut représente l'historicité, et par son contact avec Saintré transpose l'ouvrage de roman en document, et le Saintré romanesque en Saintré authentique. Ainsi aussi des listes de croisés, dûment authentifiées par l'exactitude des informations: blasons, cris de guerre. Voilà Saintré désormais "cadré" par son appartenance à une histoire qui comprend un compagnon indubitablement historique et dont la famille était toujours florissante en $1456^{48}$, et par sa participation à une croisade à laquelle les détails, sans être véridiques, ont prêté un "effet de réel " ${ }^{49}$ retentissant.

Saintré sera désormais en quelque sorte autonome : Madame ne sera plus ni sa «conseillère chevaleresque» ni sa patronne financière. Lorsqu'il reviendra de la croisade, ce sera avec une réputation de guerrier et de champion national ${ }^{50}$, non pas de chevalier servant : les deux amants, Madame et Saintré, se retrouveront cette fois sur un pied d'égalité : «La furent baisiers donnez et baisiers renduz» (JS, p. 227/19$20)^{51}$. Mais cet équilibre est, on le verra, provisoire et trompeur. Quinze mois plus tard, Saintré profitera d'une liberté qu'on ressent comme autant financière que chevaleresque et personnelle pour élaborer à son tour, et sans l'encouragement, la permis-

\footnotetext{
${ }^{46}$ Voir à la note 42.

${ }^{47} \mathrm{Je}$ pense à l'expression anglaise «truth guarantee» : petite phrase par laquelle un auteur ou un historien se rend garant de l'authenticité de son récit. Voir à ce sujet Jeanette M. A. Beer, Narrative Conventions of Truth in the Middle Ages, Genève, Droz, 1981.

${ }^{48}$ Voir à la page 183 le petit paragraphe où, mine de rien, La Sale met en rapport le Boucicaut qu'il évoque dans son ouvrage et les Boucicaut contemporains : «Ce Galias de Mantua, je croy que fut puis cellui moult renommé chevalier qui combatit a oultrance messire Jehan le Maingre» (JS, p. 183/1-3).

${ }^{49}$ Voir bien sûr l'article de Roland Barthes, «L'effet de réel», Communications, 11, 1968, p. 84ss.

${ }^{50}$ Le roi lui-même se lève pour l'accueillir à la cour : «Le roy, qui assis estoit, pour les honorer et pour la grant joye qu'il avoit, se dressa sur piez et fist un ou deux pas au devant» (WS, p. $223 / 18-20$ ).

${ }^{51}$ Il serait tentant de voir dans les paroles désobligeantes de Madame ( $«$ Sire de Saintré, quant ces dames vous avront bien festoié, au moins que nous nous [sic : lire vous?] voions a nostre tour» (JS p. 224/30-32) la preuve déjà d'une transformation de Madame. Il me semble toutefois que la parfaite entente entre l'amant et la dame, véhiculée par le paragraphe que nous citons, représente en quelque sorte le point de bascule du roman, et n'est pas donc à mettre en doute.
} 
sion de Madame, un «bien d'armes» $(J S, \text { p. } 228 / 3-4)^{52}$. Le voyant ainsi échapper à sa tutelle, Madame est furieuse: «Avez vous levee emprise et departie ça et la, sans mon sceu et congié ? Jamais tant que je vive de bon cuer ne vous ameray» US, p. 233/28-30). Saintré semble reconnaitre aussitôt la rupture d'avec le pacte, le "contrat» dont nous parlions plus haut ${ }^{53}$ : son emprise avait été présentée en termes de service :

Ma dame... du temps qu'il a que je suis vostre treshumble serf et loial serviteur, onques en moy n'eust tant de bien que pour l'amour de vous j'eusse nul fait d'armes empris, mais tous ceulz que j'ai fais et ou je me suis trouvez ont esté par voz commandemens, par voz conseils et bons advis. (JS, p. 233/7-13)

Maintenant, c'est en termes de service, et donc d'une réciprocité bafouée, qu'il exprime sa déception et sa surprise :

Helas! ma dame, veez cy pouvre nouvelle, quant pour bien faire je doy estre pugny, qui vous ay tant et si loialment servie, mis mon cuer, corps, honneur et vie pour vous obeÿr, et ores que je cuidoie en vostre service faire mon devoir, acroistre vostre grace et mon honneur, fault que je perde celle a cui je suis tant actenu? Hé! ma tresredoubtee dame sans per, aiez de vostre serviteur mercy. (JS, p. 234/1-9)

Lorsque plus tard, et revenu de cette malheureuse emprise, il retrouvera une Madame mal polie et même grossière, il fondera ses reproches ici aussi sur la réciprocité :

Hélas, ma dame, est ce a bon essiant, ou pour moy essayer, que si feible response m'avez faite, qui suis cellui qui tant vous ay amee, et suis cellui qui onques ne vous desobeÿ? (JS, p. 273/25-28)

Mais Madame a, si l'on peut dire, changé de statut. Selon une métaphore que j'ai déjà employée plus haut, elle est elle-même devenue objet de phrase. La dompna qui régissait tous les détails de sa vie et de celle de Saintré est devenue irrésolue: lorsqu'elle veut refuser l'invitation de Damp Abbé, elle se laisse convaincre avec une déconcertante rapidité ( $J S$, p. 24721-248/11) ; lorsqu'elle veut exercer sur l'homme d'église son ancienne empire, celui-ci refuse : «Madame, qui moult le prie de seoir, ne le puet faire seoir» (JS, p. 249/5-6). Plus grave encore, là où elle avait sélectionné Saintré, se gardant ainsi une autorité certaine, elle se laisse séduire, est trahie par un corps qui, linguistiquement, est comme «anatomisé» («les yeulx, archiers des cuers, peu a peu commencerent l'un des cuers a l'autre traire, et tellement que les piez.. commencerent de peu a peu l'un a l'autre touchier», JS, p. 249/26-30). La banalité de l'image ne doit pas nous induire en erreur: lorsque La Sale imagine un amour créé à part entière par «ce tres enflamé dart d'amours [qui] fiert le cuer de l'un et

\footnotetext{
${ }^{52}$ Comme la guerre «normale», une croisade réussie apportait des rançons, du butin ; voir à ce sujet Vale, War and Chivalry, p. 156ss., et Philippe Contamine, Guerre, état et société : Etudes sur les armées des rois de France, 1337-1494, Paris/La Haye, Mouton, 1972, pp. 195198.

${ }^{53}$ Voir à la note 25.
} 
puis de l'autre» (JS, p. 249/31-32), c'est d'une passation des pouvoirs qu'il s'agit ; d'autoritaire qu'elle était, Madame s'est faite passive.

Cette transformation difficilement percevable - ne serait-ce que parce que Madame a adopté un discours anti-courtois, désobligeant - est pourtant radicale, comme on le verra dans une dernière épisode, paradigmatique. Saintré, par une ruse psychologiquement fine, fait que Damp Abbé se laisse tenter par un «exploit» chevaleresque. Madame prévoit quels en sont les dangers, et elle essayera une dernière fois d'imposer à Saintré le service et l'obéissance d'usage :

Sire de Saintré, nous voulons et vous commandons que sur peine d'encheoir en nostre indignacion, incontinant tous deux vous desarmez, et se ne le faites, comme fol et cornart nous vous ferons du corps et de la vie couroucier et pugnir. (US, p. 295/21-26)

En vain : celle qui dominait autrefois son cavalier servant n'a plus aucune autorité, et Saintré a peut-être compris combien peu son loyal service sera récompensé :

Or faulse desloialle telle, telle et telle que vous estes, je vous ay si tresloialment servie longuement que onques homme puist servir et complaire a femme, et maintenant pour un ribaut moynne... vous estes deshonoree et m'avez abandonné. (JS, pp. 295/29-296/4)

La boucle est donc bouclée. Le processus ludique que Madame a entamé «faire» un chevalier, «faire» un cavalier servant - se termine sur un autre jeu, où cette fois Saintré lui-même sera le meneur. Madame a osé se mettre au centre du monde chevaleresque en exigeant ce service et cette obeissance qui doivent être l'hommage librement consenti de l'amant, et a ainsi en un sens court-circuité l'apprentissage de son amant qui, selon la tradition romanesque, se devait de se lancer dans la quête qui lui aurait conféré son identité d'homme et de héros. Dans ce curieux ouvrage, Saintré remonte à contre-courant le parcours chevaleresque : confiné trop jeune dans un monde féminin, tributaire d'un schéma archétypal qui, en fin de compte, est celui de l'ascension sociale par le mariage, il trouvera son statut de chevalier non pas en conquérant mais en écartant l'héroïne dont l'amour aurait autrement, selon les normes romanesques, constitué son ultime souhait. Cet itinéraire paradoxal trouve son point de bascule précisément dans la fraternité «virile» des croisés - fraternité dont témoignent ces longues, ces interminables listes de croisés, d'armoiries, de cris de guerre. Dernier représentant peut-être d'une idéologie qu'il a dû sentir révolue ${ }^{54}$, La Sale restitue au centre d'un monde devenu désespérément romanesque et féminisé ce qui devait être pour lui le monde pur et dur de la chanson de geste, où croisés et Sarrasins se confrontent dans la profonde conscience d'une mâle vocation guerrière.

Jane H. M. Taylor

${ }^{54}$ Que d'ailleurs son Des anciens tournois et faits d'armes laissait prévoir ; voir l'édition de B. Prost, Traicté de la forme et devis comme on faict les tournois, Paris, 1878, pp. 193-221. 